\title{
Teenage pregnancies that end in abortion: what can they tell us about contraceptive risk-taking?
}

\author{
Lesley Hoggart, ${ }^{1}$ Joan Phillips ${ }^{2}$
}

\author{
'School of Health and Social \\ Care, University of Greenwich, \\ London, UK \\ 2Policy Studies Institute, London, \\ UK
}

\section{Correspondence to Dr Lesley Hoggart, Principal Research Fellow, School of Health and Social Care, University of Greenwich, Avery Hill Campus, Southwood Site, Avery Hill Road, \\ London SE9 2UG, UK; \\ L.Hoggart@gre.ac.uk}

Material from the report on which this article is based has been presented on 13 January 2010 at a London Sexual Health Commissioners Networking Meeting, and at the London Sexual Health Commissioning Board Meeting on 22 February 2010.

Received 15 March 2010 Accepted 30 November 2010

\begin{abstract}
Background and methodology In 1999, the Government set the ambitious target of halving the number of under-18 conceptions by 2010 . It is now clear that this target will not be met. Much media and policy attention has been paid to teenage mothers, and yet approximately $50 \%$ of teenage conceptions end in abortion not motherhood. In London, where the present research was based, the percentage is significantly higher. The research into teenage abortion and repeat abortion, though based in London, generated insights that could potentially help different areas reduce the number of under-18 conceptions ending in abortion. A qualitative research methodology was adopted and a wide range of interviews were conducted with young women, and professionals, in 10 London primary care trusts. Results Our analysis adds to a substantial body of qualitative research that points to the complexity of sexual decision-making for young women. Contraceptive risk-taking was evident as some young women spoke of the difficulties they experienced with user-dependent methods (primarily the condom and the pill) in often unplanned, sexual encounters. They were also generally poorly informed about different contraceptive methods. Misunderstandings about fertility also emerged as an important issue that can lead young women to draw the wrong conclusions if they do not become pregnant following unprotected sex.

Conclusions Young people need improved access to, and informed understanding of, the full range of contraceptive methods available to them. In addition, efforts should be made to enable young women to have a better understanding of their own likely fertility.
\end{abstract}

\section{Introduction}

The Teenage Pregnancy Strategy, implemented in 1999, set an ambitious target of halving under-18 conceptions by 2010. Although pregnancy rates have

\section{Key message points}

Young people often struggle to practise 'safer' sex consistently, and many rely upon on a single user-dependent method such as the condom or the pill.

Young women may believe that they are infertile if they do not become pregnant following unprotected sex.

> Young women may be poorly informed about the availability and suitability of different types of contraception.

declined, it is now clear that this target will not be met. ${ }^{1}$ Furthermore, according to the latest available comparative statistics, the rate of teenage pregnancy in England and Wales remains the highest in Western Europe. ${ }^{2}$ Approximately 50\% of these conceptions end in legal abortion (elective termination), and there is considerable policy interest in reducing the numbers of what are generally assumed to be unintended and unwanted teenage pregnancies. ${ }^{1}$

Qualitative research that has looked at young women's contraceptive risk-taking in more detail has been dominated by work looking at conceptions that end in motherhood. ${ }^{3}$ While notions of intent are complex and uncertain, research has suggested that a proportion of such pregnancies are intended; ${ }^{45}$ and also that unintended teenage pregnancies can be, or become, wanted. ${ }^{67}$ This is more likely to be the case in localities with a cultural acceptance of teenage motherhood and non-acceptance of abortion. ${ }^{8-10}$

This paper presents some findings from qualitative research that aimed to improve the understanding of the contraceptive risk-taking behaviour of teenagers who become pregnant and then have an abortion. It adds to the evidence base needed in order to help young women exercise effective reproductive control, and thus avoid unintended and unwanted pregnancies. The research took place in London, where the percentage of under-18 conceptions 
that end in abortion was above the national figure and stood at $63 \%$ in $2007 .{ }^{11}$ The full results of the research are reported elsewhere. ${ }^{12}$

\section{Methods}

The research explored young women's attitudes towards sexual behaviour, contraception and abortion decision-making. A qualitative research methodology was adopted and the following interviews were conducted:

- 10 interviews with Teenage Pregnancy Strategy Coordinators (TPSCs)

- 14 interviews with other key informants involved in referring and/or supporting young women who become unintentionally pregnant

- 8 interviews with abortion providers

- 1 discussion group with 13 London TPSCs

- A short electronic questionnaire (open-ended questions) returned by 7 TPSCs who had not attended the discussion group

- 16 focus groups in schools (girls aged 14-16 years)

- 10 one-to-one in-depth interviews with teenagers who had terminated pregnancies (aged 16-19 years at time of interview)

- An expert deliberative focus group towards the end of the research process.

The research design gained pan-London approval from Wandsworth Research Ethics Committee in 2008. All the participants were given appropriate information leaflets, and were also given the opportunity to ask questions about the study and their involvement before being asked for written consent. Written parental consent was also gained for those under-16s who participated in the focus groups. The research team assessed whether each participant understood what they were consenting to (Fraser competence), and continued to monitor this understanding throughout the interview. Every effort was made to try and avoid any negative outcomes for the young women interviewed. The researchers also had the details of referral routes to sources of help and support and these were offered to participants at the end of the interview. The anonymity of all the participants has been preserved in this paper.

A number of measures were taken in order to facilitate reliability and validity. The data were analysed independently by the two researchers undertaking thematic analysis, ${ }^{13}$ within the overall analytical approach of analytic induction. The thematic analysis generated the categories and emerging findings, which were then tested and modified through constant comparison across the entire dataset. In particular, emerging themes from the focus groups and one-to-one interviews were contrasted and points of difference noted. Not surprisingly, the focus group data proved important in mapping out what might be considered dominant sexual and societal 'norms' for these groups of young women. Such 'norms' can be seen as part of the contexts for the individual level data from the one-to-one interviews. The data was also analysed in two different ways: the thematic analysis was undertaken by coding with the help of NVivo $8^{\mathrm{TM}}$ software [QSR International (UK) Limited, Southport, UK] and alongside this process, each transcript was re-read as a whole as part of a search for deviant cases, and alternative themes. ${ }^{14}$ This paper focuses on the data from the focus groups, and the one-to-one in-depth interviews, though it also draws on understandings developed across the dataset.

We report on central themes that are grounded in the data, and the quotes selected are designed to represent these themes rather than present the views of any one respondent. When cases, or themes, are nontypical this is noted. We have not, however, quantified these themes as no claims to statistical representation have been made, and quantification would be misleading in a small exploratory qualitative study. We would not seek to generalise from this particular study, but note that the findings presented in this paper concur with other research findings and can thus play a part in establishing a body of research with the potential for generalisation.

\section{Research findings}

It is now widely acknowledged that explanations for teenage sexual behaviour are complex and diverse, depending on individual motivations and social and cultural circumstances. ${ }^{3}$ The findings reported here focus on the relationship between sexual behaviour and young women's changing perceptions of their pregnancy risk. The research adds to a growing body of work that points out that some young women's poor understandings of their own likely fertility may perpetuate 'risky' sexual behaviour. ${ }^{15-17}$ It is an important addition to this literature because it is based on interviews with young women who had not wanted to become pregnant and, as reported elsewhere, ${ }^{12}$ were not untroubled about having an abortion. In other words, although they were taking contraceptive risks this was not because they were care-free about the prospect of having an abortion.

\section{Teenage sexual behaviour}

As noted in previous research, a complex web of influences lead to sexual decision-making being fraught with difficulties for many young people. These influences may include, for example: difficulties negotiating contraceptive use due to 'chaotic lifestyles', ${ }^{18}$ the influence of drugs or alcohol, ${ }^{19-21}$ and coercion..$^{22}$ Our research confirms this evident complexity. It also points to a tension between positing the need for controlled sexual encounters for 'safer' sex; and what Michelle Fine has referred to as "the missing discourse of desire". ${ }^{23-25}$ A growing body of work has sought to acknowledge adolescence as a period in which girls (as well as boys) explore their sexuality. ${ }^{26}{ }^{27}$ Such work calls for a more 
nuanced understanding of young women's sexual relationships in which the relationship between individual sexual desire and sexual autonomy and social and cultural contexts are considered. 252728

As might be expected, in this study the focus group data revealed the participants' understandings of the socio-sexual cultures within which teenage sexual activity occurs. What was understood as the normalisation of teenage sexual activity through mutually reinforcing messages about the desirability and excitement of teenage sex from multiple media sources, and peers, was a strong theme. In particular, the assumption that by a certain age (most often seen as over 16 years) "all their friends are doing it" was seen as a central point in deciding whether to have sex. Older girls in the focus groups talked about feeling pressured not only to maintain their relationship with boys but their own reputation as well: "that is to say, if you are not doing it something is wrong with you". This chimes with recent work that has argued that as a consequence of a greater sexualisation of culture, young women may be under pressure to show sexual autonomy and sexual knowledge. ${ }^{29} 30$

In the one-to-one interviews, however, individual choices and behaviours were also evident. A complex, and often ambiguous, relationship between expectations and desires was also evident:

"I felt like why am I following everyone else, but at the same time I wanted to do it, and at the same time it was like yeah, everyone else is doing it why not me?"

This extract illustrates the wish to exercise sexual autonomy and not simply follow patterns of behaviour laid down by others. However, in this case, the respondent's own desires were, in a sense, reinforced by the perception that "everyone else is doing it".

The older teenagers (in-depth one-to-one interviews) who had become pregnant pointed to a lack of control over many encounters, but additionally revealed an ambiguity of intentions, as demonstrated in the following explanation of unplanned first sexual intercourse: "that was a night of drunkenness. He was like my primary school crush so I kind of allowed him to get away with it". The respondent thus begins by connecting what she has experienced as a loss of control to being drunk. However, this is ambiguous as she also states that she "“allowed him to get away with it", possibly indicating that it was something that she had also desired, or at least that she had some degree of choice over, maybe because he was her "primary school crush". These extracts are illustrating a strong theme, largely absent in the focus group data, in which the relationship between sexual autonomy and expected behaviours and sexual 'norms' is expressed. These differences in the data can be partly explained by the different types of interviews, as respondents were able to explore these issues in more depth in a one-to-one interview. However, it is also worth noting that these were also older teenagers who had been sexually active for varying lengths of time, and were therefore better able to reflect on their own sexual motivations.

Analysed together, the data from focus groups and the one-to-one interviews therefore point to a complex interaction of individual teenagers working out their own wishes with respect to decisions about engaging in sexual activity in a context in which a range of influences are being felt. Further, they often present a picture of uncertainties and tensions negotiating this process. Such tensions have negative implications for the effective use of condoms, young people's preferred method of contraception. ${ }^{31}$

Many of the teenagers in the focus groups (and, to a lesser extent, in the one-to-one interviews) expressed anxieties about using condoms (the contraceptive most usually referred to), and also exhibited poor knowledge about other methods of contraception; as well as uncertainty about where to turn to for contraceptive help and advice.

The difficulty of reconciling sexual spontaneity with contraceptive planning was an additional theme in the narratives of the older teenagers (that had terminated pregnancies). When they first became sexually active, "being safe" had proved difficult to put into effect even when they were aware of the possibility of becoming pregnant. This was sometimes because they lacked the confidence (and/or were embarrassed) to ensure that condoms were used. At other times, it was because they themselves did not want to disrupt the spontaneity of sexual activity:

"I do think about it but it's difficult just to interrupt it or think, ob we've got to get that or stop it so you can get a condom and also if I don't say something the guy doesn't usually say something and it's just weird."

Such different influences are some indication of the range of issues that may affect teenage sexual encounters. Different relationships are likely to demonstrate contrasting power relations and sexual dynamics, but many factors can contribute towards making practising 'safer' sex difficult for teenagers.

In addition, it is known that some teenagers experience what was described by sexual health professionals as "chaotic lifestyles", lacking stability at home and/ or at school. In such individual circumstances, a possible unintended pregnancy is only one of their problems. ${ }^{18} \mathrm{~A}$ minority of our interviewees might have been described in this way. In common with some of the other teenagers, they had chosen to use the pill, but struggled to take it consistently.

Although they had been aware of the implications of unsafe sex, the teenagers who had unintended pregnancies had engaged in risk-taking behaviour through inconsistent use of user-dependent contraceptives. Most of them talked about initially being very worried about becoming pregnant, and a few had accessed emergency contraception occasionally when they had 
first become sexually active. However, a continuation of contraceptive risk-taking was then partially prompted by their poor understanding of fertility.

\section{Misunderstandings and misperceptions about fertility}

In the main, the teenagers interviewed, especially in the focus groups, thought it was "really easy" to become pregnant. Running alongside this view, however, was the assumption that if you had unprotected sex and did not become pregnant then you were probably infertile. Young women's own experiences thus encouraged an "it won't happen to me" approach to perceptions of pregnancy risk.

Recent findings from a study on how young people use condoms suggest that worries about becoming pregnant, rather than sexually transmitted infections (STIs), drive condom use. ${ }^{32}$ Misunderstanding pregnancy risk therefore has the potential to increase the likelihood of unprotected sexual intercourse. Such a pattern of behaviour has been identified in research on emergency contraception. ${ }^{15} 16$ This pattern was also a strong theme in this research project, evident among some of the teenagers who had become pregnant and terminated their pregnancies. Their misunderstandings about fertility followed an identifiable pattern. Contraception had generally been used during initial sexual encounters when they were worried about becoming pregnant and - crucially - thought that becoming pregnant was "very easy". However, as discussed earlier, this was often a struggle, and when instances occurred in which no contraceptive was used, or it was used imperfectly, without a resulting pregnancy, a (mis)perception emerged of being at low risk of pregnancy, or even of being infertile. This emerging belief led these young women either to stop using contraception, or use it in a haphazard manner, thinking it was unimportant. As with the research on emergency contraception they became "frequent nonusers of contraception [who] did not think that pregnancy would happen to them" (p. 313). ${ }^{15}$ Although in some cases they may have been testing their fertility, the dominant message in the narratives was that they did not need to be careful because they thought they could not get pregnant.

"I just thought that I couldn't get pregnant because I think there was one time when I was 15 and I didn't get pregnant with [boyfriend], that's probably what I thought 'I can't have babies' sort of thing."

"The few times I had had sex with him, I never got pregnant so I thought, like I said before, 'there must be something wrong with me why I can't conceive or carry a child', so that's why, that's another reason why I didn't think about contraception."

\section{Discussion}

This research adds to a growing body of qualitative work that understands teenage sexual decision-making within a context of complex, and often competing, pressures and influences. ${ }^{33} 34$ The tension between 'safer sex' necessitating an element of control and the reality of many sexual encounters was evident in the accounts of teenagers themselves.

Contraceptive service providers should therefore be aware of the difficulties that many young women face, and that this has implications for their effective use of contraception. The findings of the research also showed that young people lacked knowledge on the full range of contraceptive choices; were often unaware of how to access services; and relied on user-dependent methods. Local services therefore need to ensure that a full range of contraceptive methods - including all long-acting contraceptives and emergency hormonal contraception - are easily accessible to women of all ages, and that contraceptive consultations allow sufficient time for full discussion of appropriate methods (including possible side effects, and differences in effectiveness depending on 'normal' or 'perfect' usage). Further support may be needed to help maintenance of the selected method, or movement to another method. The persistent unevenness in local contraceptive and sexual health services that has recently been acknowledged needs further attention. ${ }^{35} 36$

The findings also have implications for the content and delivery of Sex and Relationships Education (SRE) where there is currently an emphasis on condoms. The messages for young people need to be more complex, and - crucially - separate discussions are needed about pregnancy prevention and STI prevention. All teenagers (boys and girls) should be given the opportunity to discuss the pros and cons of all methods of contraception, as well as given enough time to engage with the complexity of sexual decision-making. Young people need to be encouraged to discuss which contraceptive methods are more effective for each purpose, and to consider the possible benefits of using more than one method. Contraceptive and sexual health services should be clearly signposted in SRE.

With respect to unintended pregnancies, fertility misperceptions emerged as an important consideration; taking the focus group data and the narratives of the young women who had terminated pregnancies together it appears that difficulties around control and misperceptions about fertility work together. Given that teenagers struggle to practise 'safer' sex consistently there are almost certainly going to be instances of unprotected sexual intercourse that do not end in pregnancy. Poor awareness of pregnancy risks has been shown to militate against using emergency contraception and result in unintended pregnancies. ${ }^{16} 3738$ International research has shown similar patterns. ${ }^{39} 40$ This particular research has shown this pattern of sexual behaviour among teenagers who were certain they did not want to become pregnant.

While it is not possible to generalise from this smallscale qualitative study, it does therefore add to existing research conducted elsewhere in the UK, with a qualitatively different sample and set of research questions. 
It is becoming clear that young people would benefit from improved understanding of the unpredictability of the relationship between unprotected sex and pregnancy. Much uncertainty about pregnancy risk could be undermined if young women had a better understanding of their own bodies and did not begin to doubt their fertility following a single act of unprotected sexual intercourse.

Acknowledgements This paper draws upon research findings from a recently completed research project: Young People in London: Abortion and Repeat Abortion. The authors would like to acknowledge the work of Angela Birch of CSUK (a consultancy specialising in supporting PCTs and Local Authorities to deliver client-centred solutions to prevent teenage pregnancy and reduce the level of risk-taking behaviour in young people), who undertook some of the fieldwork for this project; and Ofra Koffman (Kings College, London) who helped with the literature review. The project was commissioned by the Young London Matters Teenage Pregnancy Work Strand of the Government Office for London, and the authors would like to acknowledge the support and guidance of the London Teenage Pregnancy Coordinators, Judy Mace and Adrian Kelly. They would also like to thank all the members of the Advisory Committee who were generous with their time and assistance. Finally, the authors would like to thank all those people who took part in this project, including all the local service providers interviewed, many of whom also helped with the planning and facilitation of the research. In particular, they thank all of the young women who gave so generously of their time and experience.

\section{Competing interests None.}

Ethical approval The study was approved by Wandsworth Research Ethics Committee in 2008.

Provenance and peer review Not commissioned; externally peer reviewed.

\section{References}

1 Department of Health. Abortion Statistics, England and Wales: 2007. Statistical Bulletin 2008/1. London, UK: Department of Health, 2008.

2 Population Action International. A Measure of Survival: Calculating Women's Sexual and Reproductive Risk. Washington, DC: Population Action International, 2007.

3 Arai L. Teenage Pregnancy: The Making and Unmaking of a Problem. Bristol, UK: The Policy Press, 2009.

4 Hek R, Hoggart L. Young Women, Sex and Choices: A Study of Young Motherhood in Shropshire. London, UK: Middlesex University, 2004.

5 Cater S, Coleman L. 'Planned' Teenage Pregnancy: Perspectives of Young Parents from Disadvantaged Backgrounds. Bristol, UK: The Policy Press, 2006.

6 Hoggart L. Young women sexual behaviour and sexual decision making. In: Growing Up with Risk, Thom B, Sales R, Pearce J (eds). Bristol, UK: Policy Press, 2007;185-202.
7 Duncan S, Edwards R, Alexander C (eds). Teenage Parenthood: What's the Problem? London, UK: The Tufnell Press, 2010.

8 Henderson LR. A survey of teenage pregnant women and their male partners in the Grampian region. Br J Fam Plann 1999;25:90-92.

9 Hoggart L, Nasserzadeh S, Shamash M. Young Women, Sex and Choices: A Study of Young Motherhood in Haringey. London, UK: Middlesex University, 2006.

10 Lee E, Clements S, Ingham R, et al. A Matter of Choice? Explaining National Variation in Teenage Abortion and Motherhood. York,UK: Joseph Rowntree Foundation, 2004.

11 Office for National Statistics. Conception Statistics in England and Wales 2007 (Provisional). Table 7: Under 18 Conceptions: Numbers, Rates and Percentage Leading to Abortion by LAD1 Area, 2007. http://www.statistics.gov.uk/downloads/theme_ health/conceptions2007/provisional_annual_conceptions_2007. xls [accessed 20 February 2010].

12 Hoggart L, Phillips J. Young People in London: Abortion and Repeat Abortion. London, UK: Government Office for London, 2010.

13 Braun V, Clarke V. Using thematic analysis in psychology. Qual Res Psychol 2006;3:77-101.

14 Silverman D. Interpreting Qualitative Data: Methods for Analysing Talk, Text and Interaction (2nd edn). London, UK: Sage Publications, 2001.

15 Williamson LM, Buston K, Sweeting H. Young women's perceptions of pregnancy risk and use of emergency contraception: findings from a qualitative study. Contraception 2009;79:310-315.

16 Williamson L. Contraceptive Careers: Young Women's Choices, Influences and Risks. Glasgow, UK: University of Glasgow, 2007.

17 Sørensen MB, Pedersen BL, Nyrnberg LE. Differences between users and non-users of emergency contraception after a recognized unprotected intercourse. Contraception 2000;62:1-3.

18 Hallgarten L, Misaljevich N. Reducing Repeat Teenage Conceptions: A Review of Practice. London, UK: Education for Choice, 2007.

19 Shoveller JE, Johnson JL. Risky groups, risky behaviour, and risky persons: dominating discourses on youth sexual health. Crit Public Health 2006;16:47-60.

20 Coleman LM, Cater SM. A qualitative study of the relationship between alcohol consumption and risky sex in adolescents. Arch Sex Behav 2005;34:649-661.

21 Mason LE, Mason LH. 'They haven’t a clue!’ A qualitative study of staff perceptions of 11-14 year-old female clinic attenders. Prim Health Care Res Dev 2005;6.

22 Barter C, McCarry M, Berridge D, et al. Partner Exploitation and Violence in Teenage Intimate Relationships. London, UK: National Association for the Prevention of Cruelty to Children (NSPCC), 2009.

23 Fine M. Sexuality, schooling and adolescent females: the missing discourse of desire. Harv Educ Rev 1988;58:29-53.

24 Fine M. Desire: the morning (and 15 years) after. Fem Psychol 2005;15:54-60.

25 Tolman D. Dilemmas of Desire: Teenage Girls Talk About Sexuality. Cambridge, MA: Harvard University Press, 2002.

26 Holland J, Ramazanoglu C, Sharpe S, et al. The Male in the Head: Young People, Heterosexuality, and Power. London, UK: Tufnell, 1998.

27 Tolman DL. Female adolescent sexuality: an argument for a developmental perspective on the new view of women's sexual problems. Women Ther 2001;24:195-209. 
28 Impett EA, Tolman DL. Late adolescent girls' sexual experiences and sexual satisfaction. J Adolesc Res 2006;21:628-646.

29 Gill R. Postfeminist media culture: elements of a sensibility. Eur J Cult Stud 2007;10:147-166.

30 McRobbie A. The Aftermath of Feminism: Gender, Culture and Social Change. London, UK: SAGE, 2009.

31 Lader D. Opinions Survey Report No. 41. Contraception and Sexual Health, 2008/09. Newport, South Wales: Office for National Statistics, 2009.

32 Hatherall B, Stone N, Ingham R, et al. The Choreography of Condom Use: How, Not Just If, Young People Use Condoms. University of Southampton, Southampton: The Centre for Sexual Health Research, 2005.

33 Coleman L, Testa A. Sexual health knowledge, attitudes and behaviours among an ethnically diverse sample of young people in the UK. Health Educ J 2007;66:68-81.

34 Arai L. British policy on teenage pregnancy and childbearing: the limitations of comparisons with other European countries. Crit Soc Policy 2003;23:89-102.
35 MedFASH. London Sexual Health Needs Assessment and Service Mapping, 2008.

36 Independent Advisory Group on Sexual Health and HIV. The Time is Now: Achieving World Class Contraceptive and Abortion Services. London, UK: Department of Health, 2009.

37 Free C, Lee RM, Ogden J. Young women's accounts of factors influencing their use and non-use of emergency contraception: in-depth interview study. BMJ 2002;325:1393.

38 Ziebland S. Emergency contraception: an anomalous position in the family planning repertoire? Soc Sci Med 1999;49: 1409-1417.

39 Jones RK, Darroch JE, Henshaw SK. Contraceptive use among U.S. women having abortions in 2000-2001. Perspect Sex Reprod Health 2002;34:294-303.

40 Larsson M, Aneblom G, Odlind V, et al. Reasons for pregnancy termination, contraceptive habits and contraceptive failure among Swedish women requesting an early pregnancy termination. Acta Obstet Gynecol Scand 2002;81:64-71. 